\title{
Towards Participatory Design of Social Robots
}

\author{
Aduén Darriba Frederiks ${ }^{1}$, Johanna Renny Octavia ${ }^{1}$, Cesar Vandevelde ${ }^{2}$, and \\ Jelle Saldien ${ }^{1,2}$ \\ 1 Ghent University, imec-mict, Miriam Makebaplein 1, Ghent Belgium info@mict.be \\ http://mict.be \\ ${ }^{2}$ Ghent University, Dept. of Industrial Systems Engineering and Product Design, \\ Graaf Karel de Goedelaan 5, 8500 Kortrijk, Belgium
}

\begin{abstract}
With an increase in research and development of social robotics and commercial robots entering the market, there is a need for design tools that enable non-experts to design, build and use customized social robots. Human Computer Interaction researchers have a rich evolving tool-set when it comes to user-centered design. To encourage the use of user-centered design techniques in early design iterations of social robots, we propose the use of Opsoro. We present a case study which exhibits participatory design sessions using a Do-It-Yourself platform to enable the creation of social robots with non-experts.
\end{abstract}

Keywords: Social robots. Do-It-Yourself. participatory design. toolkit. open source.

\section{Introduction}

Recent years have been characterized by an increased interest in robotics, and analysts have steadily pointed at robotics as one of the next big trends in technology. Gradually, robotics research has shifted its attention from robots that function within their own predefined space to robots that coexist with humans in the human's natural habitats. In conjunction, the research domain shifted from technological to a multidisciplinary form including social sciences. This new paradigm in robotics is focused on creating meaningful interactions with their contextual surrounding. This resulted in the study of social robotics, a field that is concerned with natural interaction between robots and humans. Social robots are robots that can communicate using social affordances [4] that we find intuitive, for example through body posture, facial expressions, speech, and gaze.

A coincidental trend shaping and disrupting our modern society is the revival of the Do-It-Yourself (DIY) paradigm. Contemporary DIY trends, such as the maker movement and the open source hardware movement, draw attention to the empowering feeling of making things. We should not restrict ourselves to being just consumers of technology, but that we should also become creators of technology in order to shape the world around us. These trends have been 
responsible for democratizing previously complex technology, including social robotics technology, in a creative, hands-on setting. Many engineering challenges that were considered extremely difficult in the past can now be accomplished with the help of open designs and off-the-shelf parts. However, it is not obvious to rapidly design DIY custom social robots. It generally requires a great amount of time, effort and relevant prior knowledge of the designer.

The work described in this paper is situated at the intersection of three trends: robotics, DIY and Participatory Design (PD). Our work foresees the possibility of providing a DIY platform to support the PD process when designing and creating custom social robots. This paper discusses how Opsoro, is used to facilitate the PD sessions conducted to design customs social robots, which we demonstrated in a case study. Through Opsoro as an (online) platform approach, it is expected to make social robotics technology accessible for a wider audience.

\section{Opsoro: Open Platform for Social Robots}

We have recognized an opportunity for an open DIY platform that facilitates the design, construction, and production of new social robot characters. To address the current difficulties of designing custom social robots, an open source, DIYfriendly toolkit approach called Opsoro [12] has been developed. This platform is based on an existing social robot, named Ono (Figure 1). Ono is designed in the context of robot-assisted therapy for children with Autism Spectrum Disorder (ASD). It is a low cost, open source social robot that can be made using DIY tools and techniques [14].

Opsoro contains an open-source programming, hardware, and embodiment framework. We focus on non-experts to go from a character concept to a functional social robot. Emphasizing low-cost and DIY aspects and aiming primarily at characters with animated facial expressions and limited body/limb motion. Opsoro has showed the feasibility of supporting the design and creation of social robots by using only DIY techniques and materials [13].

\subsection{Hardware}

The various components are grouped into subunits of related actuators and mechanisms, called modules (Figure 2). This has a number of important advantages. It simplifies assembly; modules can be put together outside of the body of the robot, where there is more room to manipulate components and perform assembly steps. Secondly, in case of damage, modules can be removed and replaced or repaired quickly. Thirdly, the modular architecture makes the system ideally suited for accelerating the design of new robots, as complex functionality is packaged in easy-to-use building blocks. Finally, the modules allow for upgrades and customization, enhancing the lifespan and potential usefulness of robots built using the system. Each module encapsulates the functionality of one specific facial/body feature, such as an eye or a mouth. As of yet, the modules focus on actuation only, though it is also possible to incorporate sensors. 


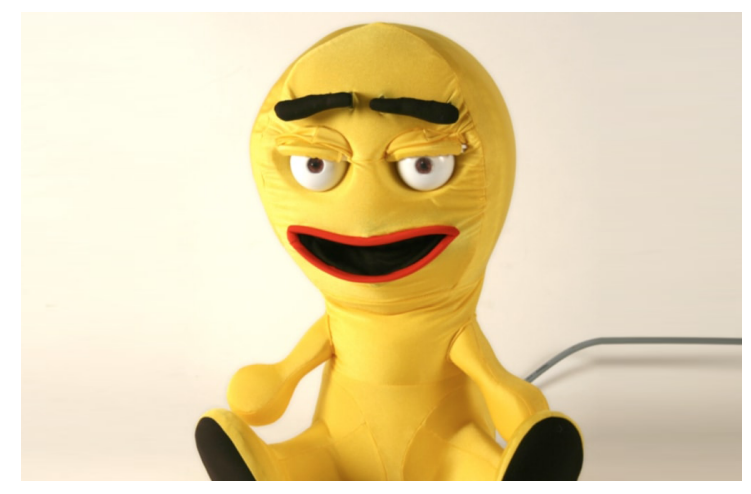

Fig. 1. Ono, the open source social robot
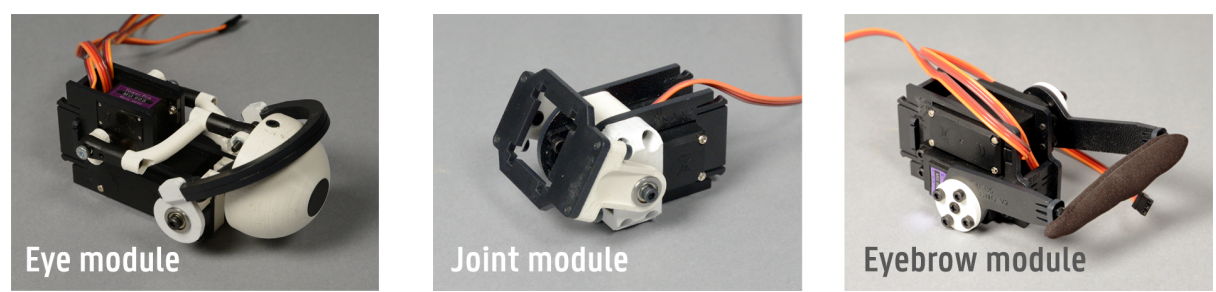

Fig. 2. Assembled Open source modules including the off-the-shelf servo motor

\subsection{Software}

The robots made with Opsoro can be controlled via a web interface running on the Raspberry Pi. The platform contains an application called Social Script to provide a very user-friendly app (Figure 3) to control facial expressions with text-to-speech. Custom programming is facilitated with the Opsoro Blockly API (Figure 4), which is a visual programming language based on Blockly ${ }^{3}$ to allow participants to program behaviors. Simple interactive scenarios can be created using this language by dragging and connecting puzzle-shaped blocks.

\subsection{Embodiment}

This design approach shares similarities with the concept of "untoolkits" [5] in the sense that the toolkit is not just a collection of modules, but that the step of creating a custom embodiment is an intrinsic part of the kit. Thus, digital manufacturing techniques such as laser-cutting can be seen as intangible components of the Opsoro toolkit. A large degree of design freedom is afforded through this approach, as the design of the modules does not have a large influence on the aesthetics and design of the robotic character. In the first approach the toolkit consisted of modules that are attached to a custom made frame [12]. This will

\footnotetext{
${ }^{3}$ Blockly - https://developers.google.com/blockly/
} 


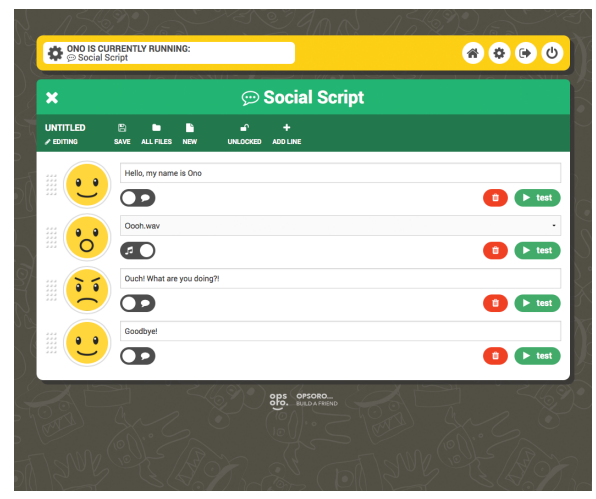

Fig. 3. Social Script App

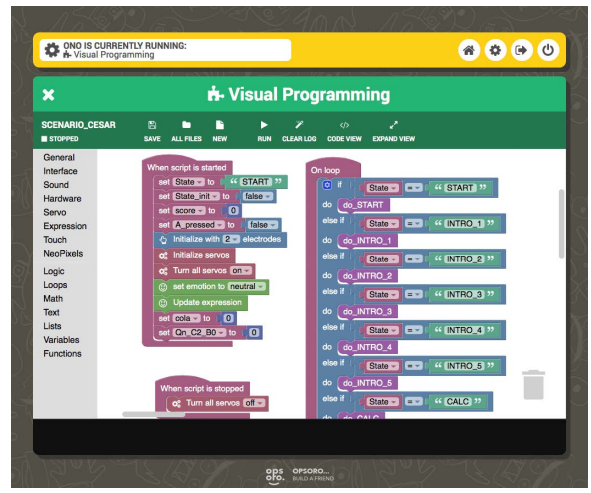

Fig. 4. Opsoro Blockly programming

serve as the skeleton of the robot where the embodiment will be fitted onto. This version allows for full freedom in customization but comes at a higher workload. For short design sessions there is a simplified version called Opsoro Grid. This will be the version used in the case study. In this toolkit the modules can be connected to a grid plate using a single screw. The holes of the grid plates are spaced $8 \mathrm{~mm}$ apart, making the system compatible with LEGO Technic bricks. The modules contain two locating pins in addition to a single threaded hole. The locating pins are used to position the module on the grid, preventing rotation. A screw is then used to fasten the module to the grid, locking the connection. Robots created using this principle are easier to produce and can be regarded as $2 \frac{1}{2}$ dimensional robots due to their flat appearance.

\section{Participatory Design in Social Robotics}

The combination of cheap off-the-shelf components and open-source software made prototyping interactive products easier and faster. What used to be highfidelity prototyping is now considered low-fidelity. Adding interactivity to a paper prototype is now cost-efficient and easy [15]. Before the rise of the DIY maker culture, designing the hardware and software was too labor intensive to be considered part of a low-fidelity prototyping stage. The introduction of paper and technology prototyping enables new paradigms in the hybrid area of PD. Beaudouin-Lafon and Mackay describe offline prototyping as a means to eliminate the constrains of modern day development tools. "Prototypes are less likely to constrain the designer's thinking ... even if the developer ends up using a standard set of interface widgets, usually results in a more creative design. " [2]. The new field of paper and technology prototyping should never restrict the offline and unlimited creativity of having just pens, paper, glue, and scissors.

Participatory Design in literature, also often referred to as co-creation or co-design, refers to the act of collective creativity of designers and people not trained in design working together in the design development process [10]. By 
applying co-creation or PD in the field of social robotics, designers and nonexperts are encouraged to creatively work together in the design and creation of social robots. In the field of human-computer interaction, PD has been widely considered to be used as a design approach or design methodology in the creation of interactive systems and applications [6].

In robotics research, there is little work within human-robot interaction that apply PD approach in the design process of creating social robots [6]. PD method has been used to elicit design recommendations, from a group of designers and target users who had a range of visual abilities, in the design process of building service robots that interact with and guide a blind person through a building in an effective and socially acceptable way [1]. A series of participatory design sessions with teenagers has also been conducted in the design of a social robot envisioned to measure their stress [8]. The use of the participatory design approach for developing social robots has also been explored in a case study with older adults diagnosed with depression [3]. Another case of co-designing social robots is with cognitively-impaired citizens, who are adults with acquired brain damages across the wide spectrum of cognitive impairments [7]. Based on these previous works, we observed two main concerns: there is a need of personalized social robots designed according to each individual's needs, and the process of co-creating such social robots can be very time consuming. The latter concern is due to a lack of practical tools that bridge the knowledge gap from both sides. Allowing both parties, research-designers and robot users, to share their knowledge through the creation of an interactive artifact. In participatory design, tacit knowledge is not only explored, it is in many cases made material [11]. In the following case study we aim to evaluate the participants confidence in their tacit knowledge for building a robot with the Opsoro system.

\section{Case Study}

As a way towards the implementation of the Opsoro platform in the participatory design of custom social robots, we carried out one design workshop as a case study to learn and observe how non-experts can build their custom DIY social robots from scratch. This workshop served as part of the iterative design process that ultimately led to the current state of Opsoro platform. To evaluate how non-experts design and build social robots from scratch using Opsoro, a design workshop was conducted during the Classroom of the Future event at the Frankfurt Book Fair. The purpose of the workshop was to evaluate the Opsoro system with secondary school students. Over the course of a two-hour workshop, the students created and programmed custom robots using cardboard, craft materials, and Opsoro modules, as depicted in Figure 5.

\subsection{Procedure}

During the workshop, participants worked together in small groups (2-5 persons per team) to design and program their robots. Four groups worked in parallel. 


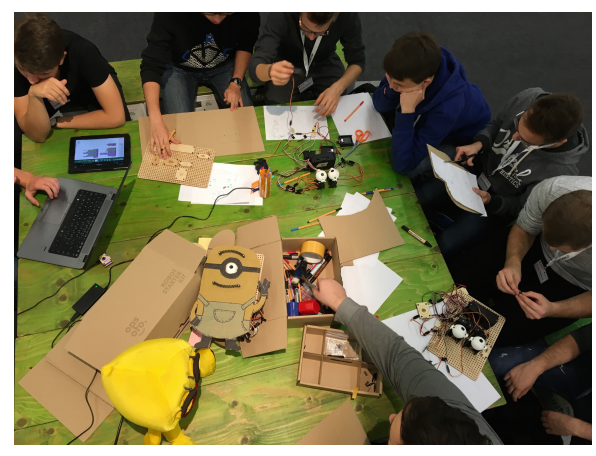

Fig. 5. Participants building custom robots using the Opsoro modules and craft materials

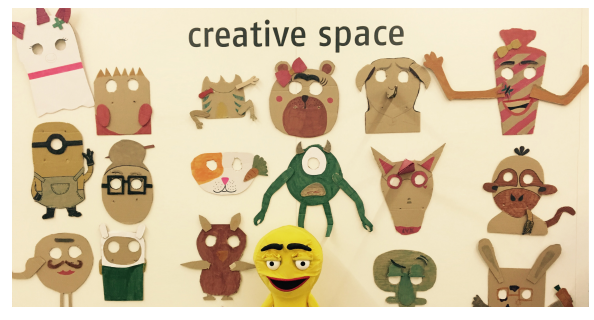

Fig. 6. Cardboard embodiments designed by the participants

The experiment ran over the course of a day, each team took approximately two hours to complete a robot. The kit consists electronics, five actuator modules, and a large grid plate. Keeping in line with the efforts to simplify and reduce costs of the Opsoro system, we chose to use smaller, simplified modules, as informed by the results of the previous workshop. The modules used during this workshop were smaller, had less DOF, and were built using micro-sized servos (as opposed to standard-sized servos). To design robots, participants first positioned and affixed the modules on the grid plate. Then, they created cardboard "skins" (Figure 6) to go over the modules and backplate. The grid system afforded participants the ability to position modules wherever they wanted. The less prescriptive design of the modules in combination with the low weight of the materials enabled functionality such as moving arms and ears.

As part of the evaluation, participants were asked to fill in a questionnaire. The large number of participants, the limited amount of time, as well as the language barrier meant that a paper questionnaire was the best evaluation tool for the workshop. The questionnaire, translated in German, consisted of the following questions:

- General personal details: name, age, and gender.

- 7-point Likert scale statements, with a value of one indicating complete disagreement, and seven indicating complete agreement:

- "I could build what I wanted to build."

- "The connection system is easy to use."

- "The modules are adaptable."

- "I like the aesthetics of the Opsoro system."

- "I like the functionality of the Opsoro system."

- "I like the novelty of the Opsoro system."

- The AttrakDiff-Short questionnaire. This variant of the AttrakDiff questionnaire consists of only ten antonym-pairs, as opposed to 28 antonym-pairs in the full AttrakDiff questionnaire. 
- Open questions:

- "Which workshop aspects did you like?"

- "What was the most annoying aspect, or where did you experience the most problems?"

- "What did you learn from the workshop?"

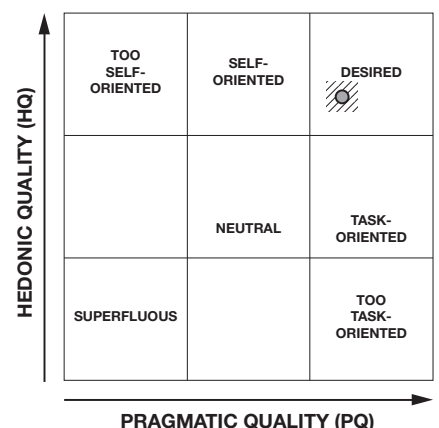

Fig. 7. Results of the AttrakDiff-Short questionnaire. The hatched area represents the confidence rectangle $(n=46, \mathrm{PQ}=1.52 \pm 0.26$, $\mathrm{HQ}=1.64 \pm 0.24)$

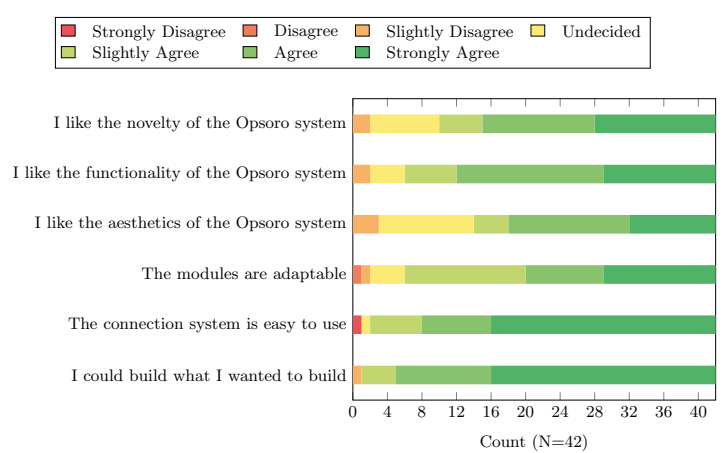

Fig. 8. Results from the Likert scale questions $(n=$ 42)

\subsection{Results}

In total, 48 workshop participants filled in the questionnaire. The average age of respondents was 17.35 years old, with a spread of $\sigma=1.86$. The youngest respondent was 14 years old and the oldest was 23 years old. Respondents were predominantly female (66\%), with 32 girls versus 16 boys. Figure 7 shows the result of the AttrakDiff-Short questionnaire and position the Opsoro Grid system firmly within the quadrant of desirable products. Two incomplete questionnaires were discarded for the AttrakDiff, resulting in a sample size of $n=46$, and six for the Likert scale questions resulting in $n=42$. The latest iteration of the Opsoro system is situated firmly in the category of desirable products, a notable improvement compared to the previous workshop. With a value of 1.84 , the overall attractiveness of the system $(A T T)$ was also rated fairly high. The large number of participants resulted in a narrower, more precise confidence interval, as indicated by the hatched area. Figure 8 shows the results of the six Likert scale questions.

In the feedback from the open questions, we noticed that "creativity" is frequently mentioned as a positive aspect of the Opsoro system. From the 41 participants that filled in the open questions, 20 mentioned "creativity" in their responses (48.8\%), 10 mentioned fun (24.4\%), and 7 mentioned technology (17.1\%). 
Multiple participants also said that building robots is not as hard as it appears, and that it is fun to combine technology with creativity. As expected, negative feedback was mostly related to technical problems, such as defective servos and problems with the audio quality and the volume of the speaker. Comments from the open questions also hint at the potential of Opsoro for the Science, Technology, Engineering and Mathematics (STEM) education, as indicated by remarks such as "Technology does not have to be boring" and "You can do many cool things with technology".

\section{Conclusions}

This paper presented a case study describing the participatory design process of creating custom social robots by using Opsoro, an open platform for DIY social robots. The platform enables designers and non-experts to design, build, and use custom social robots for face-to-face communication. Opsoro provides social robotic technology to a wide audience of users. It builds upon contemporary DIY principles and practices e.g. the open source hardware movement and the maker movement. Designing a platform is different than designing a single system, not only from a technical standpoint, but in the way they are used. For this reason, traditional engineering paradigms were eschewed in favor of an iterative, usercentered design process that emphasizes user experience aspects. Each iteration has led to a better understanding of different DIY approaches in the design, build, and use phases of the platform. The result is an inexpensive, open source, DIY-friendly platform for the design, development and use of custom emotionally expressive robotic characters. The platform lends itself for participatory design sessions early in the development process of a social robot and it can serve as a toolkit for educational purposes.

Using a participatory design approach early in the development of a social robot can be advantageous for embodiment- and interaction design. The design iterations for Opsoro have led to the simpler form named Opsoro Grid that shows great potential as a design tool. The ability of taking the concept out of the lab environment and evaluate it with stakeholders through participatory design methods has already been proved valuable in the HCI community [3,7-9]. Having the right tools with these methods will open up new possibilities for

robot design research and development. Opsoro adds physical robot modules to the design toolbox for the future researchers.

\section{References}

1. Azenkot, S., Feng, C., Cakmak, M.: Enabling building service robots to guide blind people: A participatory design approach. In: The Eleventh ACM/IEEE International Conference on Human Robot Interaction. pp. 3-10. HRI '16, IEEE Press, Piscataway, NJ, USA

2. Beaudouin-Lafon, M., Mackay, W.E.: Prototyping tools and techniques. In: Jacko, J.A. (ed.) The Human Computer Interaction Handbook, chap. 47, pp. 1081-1104. CRC Press, Boca Raton, FL, 3 edn. (2012). 
3. Lee, H.R., Šabanović, S., Chang, W.L., Nagata, S., Piatt, J., Bennett, C., Hakken, D.: Steps toward participatory design of social robots: Mutual learning with older adults with depression. In: Proceedings of the 2017 ACM/IEEE International Conference on Human-Robot Interaction. pp. 244-253. HRI '17, ACM, New York, NY, USA (2017).

4. McArthur, L.Z., Baron, R.M.: Toward an ecological theory of social perception. Psychological Review 90(3), 215-238 (1983).

5. Mellis, D.A., Jacoby, S., Buechley, L., Perner-wilson, H., Qi, J.: Microcontrollers as material: crafting circuits with paper, conductive ink, electronic components, and an untoolkit. In: Proceedings of the 7th International Conference on Tangible, Embedded and Embodied Interaction. pp. 83-90 (2013).

6. Muller, M.J.: The human-computer interaction handbook. chap. Participatory Design: The Third Space in HCI, pp. 1051-1068. L. Erlbaum Associates Inc., Hillsdale, NJ, USA

7. Rodil, K., Rehm, M., Krummheuer, A.L.: Co-designing social robots with cognitively impaired citizens. In: Proceedings of the 10th Nordic Conference on HumanComputer Interaction. pp. 686-690. NordiCHI '18, ACM, New York, NY, USA (2018).

8. Rose, E.J., Björling, E.A.: Designing for engagement: Using participatory design to develop a social robot to measure teen stress. In: Proceedings of the 35 th ACM International Conference on the Design of Communication. pp. 7:1-7:10. SIGDOC '17, ACM, New York, NY, USA (2017).

9. Šabanović, S., Chang, W.L., Bennett, C.C., Piatt, J.A., Hakken, D.: A Robot of My Own: Participatory Design of Socially Assistive Robots for Independently Living Older Adults Diagnosed with Depression. In: Lecture Notes in Computer Science (including subseries Lecture Notes in Artificial Intelligence and Lecture Notes in Bioinformatics), vol. 9193, pp. 104-114 (2015).

10. Sanders, E.B.N., Stappers, P.J.: Co-creation and the new landscapes of design. CoDesign 4(1), 5-18 (2008).

11. Spinuzzi, C.: The methodology of participatory design. Technical communication 52(2), 163-174

12. Vandevelde, C., Saldien, J.: An Open Platform for the Design of Social Robot Embodiments for Face-to-Face Communication. In: Proceedings of the 11th International Conference on Human-Robot Interaction. pp. 287-294. Christchurch, New Zealand

13. Vandevelde, C., Saldien, J.: Demonstration of OPSORO - an open platform for social robots. In: 2016 11th ACM/IEEE International Conference on Human-Robot Interaction (HRI). pp. 555-556. No. 1, IEEE (mar 2016).

14. Vandevelde, C., Saldien, J., Ciocci, C., Vanderborght, B.: Ono, a DIY open source platform for social robotics. In: Butz, A., Greenberg, S., Bakker, S., Loke, L., De Luca, A. (eds.) Proceedings of the 8th International Conference on Tangible, Embedded and Embodied Interaction

15. Zhu, K., Zhao, S.: Autogami: a low-cost rapid prototyping toolkit for automated movable paper craft. In: Proceedings of the SIGCHI conference on human factors in computing systems. pp. 661-670. ACM 